\title{
An ontology of psychedelic entity experiences in evolutionary psychology and neurophenomenology*
}

\author{
MICHAEL JAMES WINKELMAN** \\ Retired, School of Human Evolution and Social Change, Arizona State University, Tempe, AZ, USA
}

(Received: November 14, 2017; accepted: January 18, 2018)

\begin{abstract}
Background and aims: Psychedelic entity experiences are examined from perspectives of evolutionary psychology and neurophenomenology. Their similarities with other entity experiences illustrate the need for a general biological explanation of entity experiences. Mechanisms are proposed to involve innate modules, operators, and intelligences that underlie ordinary cognitive inferences and provide the basis for supernatural thought. Methods: Comparisons of ayahuasca and dimethyltryptamine (DMT) entity experiences with other types of entity experiences show their fundamental similarities to conceptions of spirit guides, mythological beings, divinities, extraterrestrials, angels, celestial beings, demons, gnomes, dwarfs, elves, and others. Entities exemplify the properties of anthropomorphism, exhibiting qualities of humans. Comparative methods are proposed to identify common features and differences in psychedelic and other entity experiences. Results: Features of psychedelic entities reflect the functions of principal innate operators and modules (i.e., animacy detection, social role inferences, and mind reading) that have central roles in the explanation of the genesis of spirit experiences and beliefs. Humans' innate psychology includes diverse forms of self and alien self-phenomena, providing mechanisms for explaining psychedelic entity experiences. Neurophenomenological approaches illustrate that the physiological effects of psychedelics can account for release of innate modules and mental organs. The concept of the phantasy mode of consciousness provides a mechanism through which our unconscious causal and explanatory mechanisms produce accounts of encounters with non-human beings. The extensive interaction of DMT with the receptorome explains why these experiences give such a powerful sense of ontological certainty. Conclusion: Psychedelic entity experiences share central features with a robust innate human tendency to attribute agency, intentionality, causality, and personhood and to create accounts involving human-like qualities and entities.
\end{abstract}

Keywords: psychedelic entities, ontology, evolutionary psychology, innate modules

\section{INTRODUCTION}

What are we to make of reports of psychedelic entities, experiences of autonomous beings often experienced on psychedelics? Many reports of psychedelic entity experiences allege that they are not a hallucination nor merely some phenomena of experience, but rather a manifestation of some transcendent real noumenon involving communicative contact with another sentient being (e.g., see Luke, 2011; Meyer, 1994, 2010; St John, 2015). What is the reality of these experiences? How do we understand the ontology and origin of entity experiences, if we do not accept that they are reflections of a transcendent reality?

If we dismiss these experiences as irrelevant hallucinations without substance or meaning, we exclude significant information regarding the nature of the human mind. Yet, if we simply accept the phenomenological experiences of entities as transcendent realities, we commit an error of epistemological naivety. The Kantian distinction between phenomena and noumena is useful here. Do these psychedelic entities represent noumena, manifestations of a real transcendent reality, or are they merely phenomena produced by our complex brains, but ultimately nothing more than dream-like hallucinatory experiences?

The experience of an entity does not mean that what we see is an actual reality. The tendency for the mind to play tricks is easily illustrated in visual illusions and with distorted figures that use irregular line orientations to force the eye into a perception of movement. Various visual illusions (Figure 1) do not move as we perceive them to, but rather are distortions produced by our brain and visual system.

Nonetheless, the distinction of phenomena justifies an acceptance of the reality of the experiences for the person. Accepting the phenomenal contents of experience as data for scientific exploration of the phenomena of the human mind

\footnotetext{
*The ideas for this paper were stimulated by an invitation from Anton Binton and David Luke to participate in the Tyringham Initiative in May 2017.

** Corresponding address: Michael James Winkelman; Caixa Postal 62, Pirenopolis, Goias, 72980-000 Brazil; Phone: +1 623239 1662; +556299647 4169; E-mail: michaeljwinkelman@gmail.com
}

This is an open-access article distributed under the terms of the Creative Commons Attribution-NonCommercial 4.0 International License, which permits unrestricted use, distribution, and reproduction in any medium for non-commercial purposes, provided the original author and source are credited, a link to the CC License is provided, and changes - if any - are indicated. 


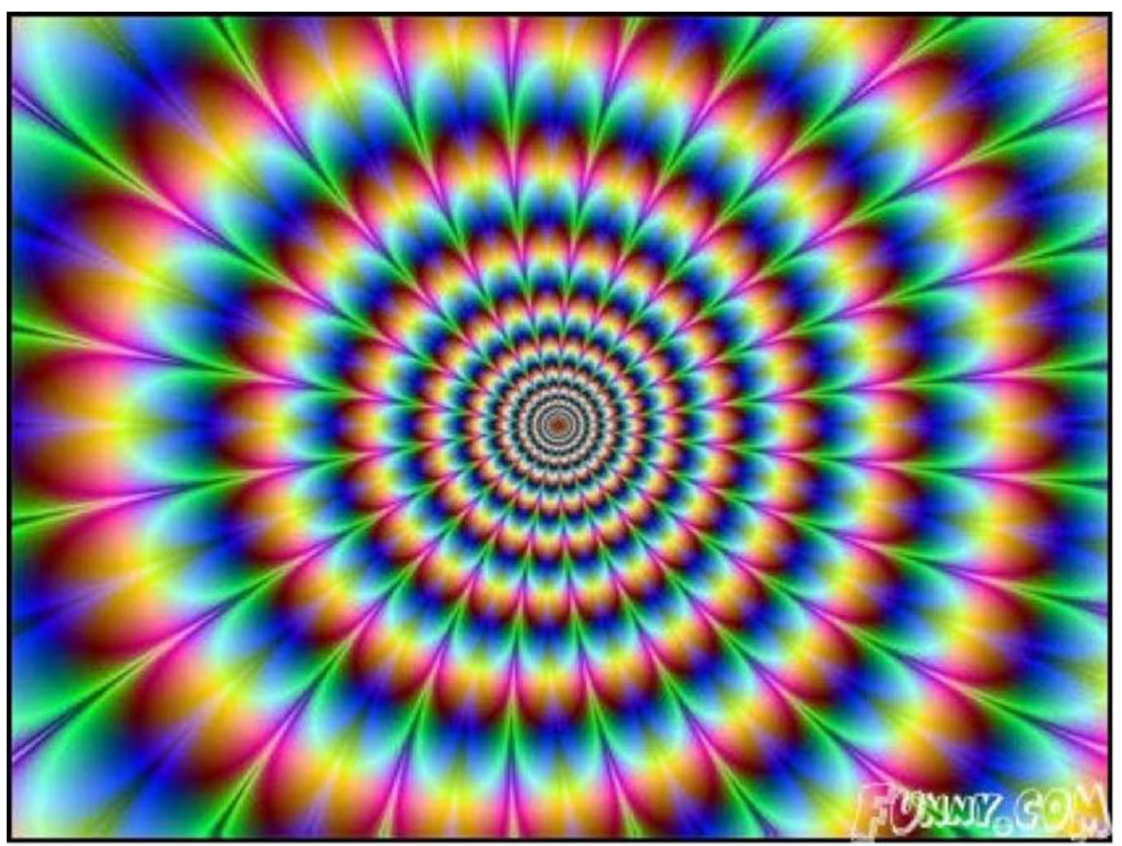

Figure 1. Illusionary pulsation

provides an empirical foundation for characterizing the nature of these experiences. Analysis of these descriptions can provide data to determine if there is an objective (intersubjective) reality to these experiences; examine their nature as conceptual entities; and identify the mechanisms producing these experiences in terms of known effects of psychedelics on brain function, potentially explaining the processes producing these often convincing manifestations.

\section{The ontological realities of psychedelic entities}

Psychedelic entities are obviously experiences that occur. How are we to conceptualize this data with respect to ontology concerned with the nature of reality and the similarities and differences among basic categories of being that may exist (Encyclopedia of Philosophy)? The MerriamWebster Dictionary's (2017) definitions of entities help to clarify at least three types of psychedelic entities: transcendent entities (noumenon, "la: being, existence; especially: independent, separate, or self-contained existence"); and objective (intersubjective phenomenon) and conceptual entities (" 2 : something that has separate and distinct existence and objective or conceptual reality").

The meaning of definition 1 - "independent, separate, or self-contained existence" - implies an empirical entity with transcendent status. True believers who allege that psychedelic entity experiences are real represent the notion of a separate and independent existence, apart from our imagination - a transcendent entity that implies a noumenon. In my view, such claims have not been substantiated with rigorous methodologies (i.e., parapsychological research). I shall leave the evaluation of this transcendent reality of psychedelic beings for others and instead address the concepts of an objective entity (intersubjectively validated experience) and conceptual entity (an explanation for such experiences).

The concepts of an objective psychedelic entity can be derived from similarities in individual experiences that point to a shared intersubjective reality underlying the experiences - are there repeatedly encountered features/ qualia in psychedelic entity encounters? The question of an objective psychedelic entity is concerned with whether across observers there are common features. Can we agree objectively - interpersonally and intersubjectively, and ultimately scientifically - about whether there are regular features exhibited in these experiences?

If encounters with psychedelic entities produce a repeatable pattern of experiences across people, those patterns establish an objective psychedelic entity, as well as a phenomenon to be explained as a conceptual entity. Just as we explain the experience of rainbows as your physical perspective on the sunlight reflecting off of water droplets in the air, we can seek naturalistic explanations of the nature of psychedelic entity experiences that do not require evoking the notion of a transcendent noumenon. There is not really a rainbow where you perceive one, but we can explain this repeatable intersubjectively validated observation through a conceptual explanation involving an understanding of the physics of light and perception.

I believe that the evidence available allows us to provide a similar materialist explanation of psychedelic entity experiences within the known frameworks of psychedelic effects on the brain. But the development of a rational and empirical discourse on psychedelic entities faces challenges due to the lack of formalized attempts at rigorous comparative examination. As Gallimore and Luke (2016) and Luke (2011) noted, we need an academic study of entity encounters that offers a thorough examination of the similarities in independent reports by identifying the recurrent characteristics common to these experiences.

This paper proposes a cross-cultural and interdisciplinary study of the features of entities and uses case materials and previous profiles of psychedelic entity experiences to offer hypotheses regarding what might be revealed by such systematic evidence. This paper outlines a methodology for 
empirically addressing the question of qualities of objective psychedelic entities and proposes a series of hypotheses regarding the qualities of these experiences based on principles of evolutionary psychology and the neurophenomenology of psychedelic experiences. These perspectives derived from evolutionary psychology provide hypotheses regarding the qualities of objective psychedelic entities, and explanations of their qualities through a conceptual definition of psychedelic entities by reference to brain processes involving innate modular structures and their functions. I propose explanations as to why objective psychedelic entities should reflect the modular structures of the brain and present a series of hypotheses derived from evolutionary psychology regarding the basic features of objective psychedelic entity experiences.

The possible qualities of objective psychedelic entities are suggested by case comparisons of reports of these experiences; this also reveals a wide diversity across various psychedelic entity experiences, including those from individuals using the same drug. Such comparisons call into doubt the assumption of a single unambiguous objective concept of a psychedelic entity experience, with a common set of features manifested across all experiences. But only through a systematic and comparative analysis of entity experiences can we determine, if psychedelic-induced entity experiences constitute a singular objective entity, a clear class of experiences that are uniquely and reliably associated with psychedelic experiences, or whether they are but another example of a widely distributed experience of various forms of an encounter with an alien other.

Nonetheless, in either case, the existence of a naturalistic basis for psychedelic entity experiences is suggested by reports of psychedelic entities that resemble other kinds of supernatural entities, such as elves, spirits, and extraterrestrials.

These similarities indicate a need for a broader explanation of psychedelic entities within the context of other kinds of mythological, spiritual, religious, and supernatural entities, an explanation found in their common basis in human biology. The apparent similarities in psychedelic entities and various other types of entity experiences found across cultures, time, and diverse conditions for altering consciousness suggest that an explanation be sought within innate functions of the human brain.

These cross-cultural similarities are examined within the context of humans' innate operators and innate intelligences (see Gardner, 2000), ancient human adaptations that provide basic aspects of our unconscious functional processing modules. These involve innate functions for specific operations, such as detecting an agent, recognizing animal species, perceiving the thoughts of social others, imitative interpretation of others (mirror neuron inference), and other adaptive automatic information processing capacities acquired in the course of human evolution.

These features of psychedelic entities involving innate intelligences have been evoked as explanations for the universality of spiritual beliefs. These reflect the operation of innate intelligences, modules, and operators for unconscious processing of the most significant features of the environment - animals and other humans (see Gardner, 2000). Our innate evolved psychology involves a number of predispositions and interpretative structures provided by biology to detect features of human-like entities. This tendency is a consequence of millions of years of adaptations for the most important factors affecting human survival - other human-like entities. These innate structures cultivated a disposition toward interpreting the external world in terms of the presence of others and their desires, intentions, temperament, etc. These tendencies reflect the necessity for adaptation to a social world in which the ability to understand the internal dispositions of other members of our species - their perceptions, thoughts, intentions, roles, personalities, evaluations, and emotions - played a crucial role in adaptation and survival.

The inevitably of projecting human-like qualities in our perception of the world requires that we assess experience of psychedelic entities with reference to our evolved predisposition to project human-like entities with certain features. The paper proposes testable hypotheses derived from evolutionary psychology that link features of psychedelic entity experiences to functions of innate modular structures of the brain.

\section{Psychedelic entopic imagery as innate modules}

A justification for evaluating the structure of psychedelic entity experiences in relation to innate brain structures is found in the phenomena of entoptics, i.e., the visual phenomena produced by innate functions of the brain. Recognition that psychedelic substances stimulate innate representational systems goes back almost a century (see Carr, 1995). In his studies of the subjective effects of mescaline, Kluver (1928) found his subjects reported recurring geometric patterns. He used this subjective data to provide characterization of the recurrent features of these entoptic images, which he labeled "form constants." The principal types he recognized included basic geometric forms; a lattice structure manifested as grating, honeycombs, and cobwebs; and openings such as tunnels, funnels, and cones.

The widespread manifestation of these innate forms includes their natural manifestations in non-drug experiences and in medical conditions (i.e., epilepsy, insulin hypoglycemia, delirium from fever or infections, and psychotic episodes); during near-death experiences and hypnagogic states; and under conditions of flickering lights, sensory deprivation, and rhythmic drumming (Siegel, 1977). The elemental forms of the entoptic images are also integrated into larger geometric patterns that typify the complex imagery of psychedelic experiences.

The central explanatory framework proposed for accounting for the features of psychedelic entities involves similar psychedelic effects stimulating (or releasing) innate aspects of neurotransmission, brain function, and the innate modular functions of the brain. These neurophenomenological explanations have the potential to highlight how psychedelic entity experiences can result in the compelling appearance of an objective reality, filled with an experiential force that results in a perceived epistemological and ontological significance.

\section{METHODS: A COMPARATIVE EXAMINATION OF ENTITY EXPERIENCES}

Do we know what are the essential and necessary features of a psychedelic entity experience - beyond that a psychedelic 
and an entity are involved? Is there a specific profile to psychedelic entity experiences, a common set of features to these entity experiences that regularly occur under psychedelic influences? Yet, there is no systematic evidence establishing such a singular experience of an objective psychedelic entity and its qualities; on the contrary, case examples show that psychedelic entity experiences take a considerable variety of forms. This paper uses case materials and others' profiles of various psychedelic entity and other entity experiences to identify the possible qualities of objective psychedelic entities and as a basis for hypotheses regarding what might be revealed by a formal cross-cultural and interdisciplinary study of such systematic evidence.

\section{Case evidence regarding objective psychedelic entity experiences}

The paintings of Pablo Amaringo (see Luna \& Amaringo, 1999) represent a person's psychedelic entity experiences. Amaringo's work highlights elements that suggest more than a single profile for such entities. Nonetheless, they generally appear as humanoids, but these reflect a variety of cultural and religious themes, including Amerindian, Spiritist, Asian, and Christian. Amaringo's depictions cover the range of themes reported by Shanon (2010) in his analysis of a wide range of supernatural beings experienced under the influence of ayahuasca. Shanon specified these different types as involving mythological beings; divinities and semi-divinities; half-human and half-animal shapeshifting hybrids; extraterrestrials - angels and celestial beings; and demons and other entities of death.

These predominantly anthropomorphic figures are mirrored in Luke's (2011) summary of various studies on dimethyltryptamine (DMT) entity experiences that reported entities characterized as gnomes, dwarfs, elves, imps, goblins and other forms of "little people," as well as angels, spirits, and gods. But even this humanoid form is exceeded in the characterizations of DMT entity experiences in modern clinical settings; Strassman's (2000) participants experienced various types of "beings," including "entities," "guides," and "aliens," but appearing similar to insects, bees, cacti, clowns, mantises, reptiles, spiders, and stick figures.

These admittedly unsystematic assessments of psychedelic entities suggest that there is no single profile for a psychedelic entity, but a diversity of appearances manifested in these experiences. Whether they nonetheless share functional properties or features in their manifestations in human experience has not been determined through systematic study of accounts. Do psychedelic entities appearing as bees, reptiles, and spiders do the same things as those appearing as elves, angels, and ETs?

Case data show that what is reported about psychedelic entities is also found in many experiences and cultural traditions, which are often evoked in describing the nature of psychedelic entity experiences. Casual comparisons show overall similarities of psychedelic entity experiences with entity experiences in other contexts, such as religious and spiritual visions, shamanism, experiences of possession, spirit allies, guardians, and animal transformation; out-of-body experiences and a range of other anomalous body phenomena; experiences of haunting, ghosts, and apparitions; encounters with various entities conceptualized in folklore and mythology traditions as dwarfs, elves, "little people," demons, etc.; experiences of unidentified flying objects (UFOs) encounters; the phenomenological content of dream experiences; and the phenomenology and neurophysiology of many psychiatric conditions, including hallucinations and properly speaking.

Whatever their unique properties may be, there are nonetheless notable parallels of many psychedelic entity experiences with other experiences of encounters with entities. For example, the experiences that result from extensive meditative practices may include encounters with entities that parallel the reports for psychedelic-induced experiences. Meditative traditions have numerous reports of experiences of entities that are encountered during profound meditative experiences, or even in everyday life!

Hancock (2000) noted substantial similarities of DMT entity experiences with experiences of fairies, UFO contact, and shamanic practices. Such similarities have profound implications for questions regarding the possible nature of objective psychedelic entities.

We need to verify if any types of entity experiences are unique to psychedelics. Are there consistent features of psychedelic entity experiences that are found only with psychedelics, or do the psychedelic entity experiences reflect the same basic properties found in entities experienced in other contexts? If there is not one type of psychedelic entity experience but a variety of subtypes, then we have several objective phenomena to be explained. If major features of psychedelic entity experiences mirror those of other kinds of entity experiences, then our questions are more broadly concerned with the explanation of entity experiences in general, as well as why psychedelics are so powerful in inducing these experiences that also occur in other contexts.

\section{Entitiology}

I propose that to determine whether there are consistent and unique features of psychedelic entity experiences, we need a cross-cultural and interdisciplinary assessment of phenomenological reports of diverse types of experiences of entities (i.e., see Winkelman, 1992). Formal quantitative comparisons of the reported characteristics of diverse entity experiences are necessary to discover any commonalities to psychedelic entity experiences and their uniqueness with respect to other types of entity experiences. We need a new field of scientific inquiry, entitiology, i.e., the study of entities, to address the questions of the nature of psychedelic, and other types of entity experiences. This field of entitiology might be viewed as partially subsumed within the field of philosophy called ontology [The Encyclopedia of Philosophy (2017) characterized ontology as concerned with the study of beings and their nature. Ontology is characterized as concerned first with the nature of being and reality, what it is that exists and is what it is made of, what are the general features of these things; ontology includes a concern with identifying the basic categories of being, determining evidence regarding what entities may exist, and how such entities may be related within a hierarchy, and subdivided according to similarities and differences (summarized from https://plato.stanford.edu/entries/logic-ontology; also see Kenny, 2012)]. 
To determine the significant features of the experience of entities, psychedelic and otherwise, we need to be able to code variables from detailed accounts of these personal experiences and subject the empirical data to systematic analysis to determine the patterns of characteristics. This assessment of the significant features of entity experiences requires first, a structured solicitation and coding of descriptions of these experiences; second, an analysis of the similarities and differences among entity experiences, by procedures such as cluster analysis which define similar groups; and finally a comparison of those empirically derived groups and their common features with other data, including the a priori labels for the experiences; the known effects of psychedelics on brain and perception; and the functions of innate modules hypothesized to account for their features.

This inquiry into psychedelic entity experiences must include entity experiences beyond those directly linked to psychedelics (as important as a clear delineation of the category "entities" might be, I do not think that it is necessary to have an a priori definition. We need to include all possible types of experiences and reports in our pool of entity data. We should be dissuaded by a priori determination or definition of entities, allowing the characterization to be empirically driven, derived from the data rather than used to define the data to be collected. While one might object that some types of experiences might not be valid for inclusion in the field of entities, empirical analyses should be able to show that the inclusion of specific kinds of data either distorts solutions or significantly increases dimension or variance in models. Inclusion of non-entity data points might also be useful in showing the relationship of entity types to non-entity concepts, providing bridges into the realm of ordinary phenomena of consciousness). If we are to establish that there are unique features of psychedelic entity experiences that require explanation, it is through the comparison of a consistent profile of psychedelic entity experiences with entity experiences that occur under other conditions. The possible unique nature of psychedelic entities cannot be effectively assessed apart from broader comparisons with all kinds of entities, with their similarities and differences providing crucial data for revealing the possibility of an objective psychedelic entity experience and its natures.

Our data on entities must be inclusive in many senses. Do entity experiences require a sense of a presence outside of oneself, exterior to the body? Or can entities also be experienced inside of the body or even just in the mind, such as in interdimensional experiences or possession phenomena? Recognition that our experience of the external world is a model produced in the mind undermines any rigid distinction between perception of internal and external entities, but such differences in experiences should be part of the data we collect from entity reports. Furthermore, psychedelic entity experiences are typically conceptualized as internal to the mind, rather than in the external world, requiring that all forms of internal entity experiences be considered for comparative purposes.

Since entities can be purely mental experiences, the experiences of dreams may also involve entities, especially incubus or succubus experiences, where there is a clear sense of being attacked by an entity in sleep. The experiences of other beings occurring during dreams provide a prototype of the entity experience, and dreams provide a normal and natural framework for assessing the possible unique qualities of psychedelic entity experiences. Indeed, the qualities of entities in dreams may manifest many, if not all, of the principal qualities of psychedelic entity experiences. On the other hand, if there are differences between psychedelicinduced and dream experiences of entities, this can reveal what is special about the psychedelic entity experiences.

Consequently, entitiology must encompass a number of existing areas of inquiry and by necessity will incorporate at least a part of the domain of the entities reported in the following areas of study:

Angelology,

Demonology,

Spiritology,

UFOology,

Folklore and Mythology studies of elves, fairies, dwarfs, pixies, imps, gnomes, goblins, leprechauns, little people, and similar phenomena reported in cultures around the world,

Possession, Mediumship, and Shamanism,

Ghosts, apparitions, and poltergeist phenomena,

Psychiatric syndromes, especially abnormal body syndromes and experiences such as the "Old Hag" and other terrorizing dreams.

A systemic coding and analysis of the features of these various accounts can determine whether or not a single type or several types of psychedelic entity experiences occur. And only through comparison with profiles obtained for reports of what are conceptualized as angels, fairies, extraterrestrials, and shamanic spirits can we determine, if there are unique features of psychedelic entities.

The data coded can include the labels given by percipients (i.e., elf, extraterrestrial, monster, etc.), but the data need to emphasize descriptions, characteristics seen or inferred such as physical features, behaviors, intentions, activities, as well as the set and setting of the percipient and entity.

Some of the general areas for coding of descriptive data regarding entities would include:

Appearances (shape, color, features, relative size to human, and other objects present);

Humanoid versus non-humanoid, animal or machine forms;

Behaviors by percipient and entity;

Intentions inferred;

Forms of communication;

Content of messages received;

Personal meanings for percipient;

Emotions expressed by entity and evoked in percipient; Agency versus mechanical;

Physical versus non-physical appearances;

Type of consciousness altering conditions present for percipient;

Types of drugs and dosage;

Non-drug alterations of consciousness - night time, isolation, chanting, prayer, fasting, etc.;

Manifestations of innate modular intelligences, brain functions, or operations - by entity and percipient.

The variable list used should be derived from grounded research as well as theory. Ideally, literature from the 
numerous areas that have already assessed the specific qualities and characteristics of various types of entities (i.e., elves, leprechauns, angels, extraterrestrials, etc.) would be extracted and used as the basis of the descriptive variables coded. We need to know how psychedelic entities appear and are experienced, as well as what they are not like. The summary of the features of angelology (Fox \& Sheldrake, 2014) is an example of preexisting variable areas ideal for such an inquiry.

The sequencing of features in the experiences should be a part of the data. Ideally, some of the personal features of respondents are also assessed, using standardized assessments [this can employ instruments, such as the Phenomenology of Consciousness Inventory (Pekala, Steinberg, \& Kumar, 1986); the Five Dimensions of Altered States of Consciousness (Studerus, Gamma, \& Vollenweider, 2010); and the Abnormer Psychischer Zustaende (Dittrich, 1998; Dittrich, Von Arx, \& Staub, 1981, 1985)] of the personal tendency for experiences of alterations of consciousness.

\section{Hypotheses regarding psychedelic entity experiences}

A cursory examination of reports of diverse entity types suggests the following hypotheses for this study.

Hypothesis 1: There is not a single type of psychedelic entity experience, but a number of different subtypes of psychedelic entity experiences.

Hypothesis 2: The distinguishing features of different subtypes of psychedelic-induced entity experiences reflect the central features of profiles of experiences reported for other types of entities.

Hypothesis 3: People reporting psychedelic entity experiences will have higher than mean scores on standard measures [this can employ instruments, such as the Phenomenology of Consciousness Inventory (Pekala et al., 1986); the Five Dimensions of Altered States of Consciousness (Studerus et al., 2010); and the Abnormer Psychischer Zustaende (Dittrich, 1998; Dittrich et al., 1981, 1985)] of susceptibility to alterations of consciousness, dissociation, hypnotic susceptibility, etc.

Determining an objective psychedelic entity phenomenon reveals the qualities to be explained, providing a basis for determining what is involved in the conceptual psychedelic entity. If the converging data from different cultures and types of entities point to common features of an objective entity, this would point to something that transcends the human cultural situation. This is found in the relationships that manifest something beyond the appearance that we perceive, whatever are the common principles of psychedelic entities beyond their obvious diversity.

\section{An example of "entity studies": The Census of Hallucinations}

These relationships among features that systematic study of the qualities of psychedelic entity experiences might reveal are illuminated by the findings of the classic 19th century Census of Hallucinations (Sidgwick, Johnson, Myers, Podmore, \& Sidgwick, 1894). This survey study asked people, "Have you ever, when completely awake, had a vivid impression of seeing or being touched by a living being or inanimate object, or of hearing a voice; which impression, so far as you could discover, was not due to any external physical cause?" Seems like a possible entity experience!

Their findings may also contribute to our understanding of entity experiences. Principal features of those entity experiences involving deaths were also generally characterized by their occurrence after awakening from sleep; a vision of a family member; and information that they had suffered a life-ending event, which was later verified. This seems to tell us something about the causal nature of these specific entity experiences - the prior condition of being in a sleep-induced alteration of conscious, an image of a close family member emerging from the unconscious, and their death, which was later confirmed. Welcome to the world of supernatural entities!

\section{HUMANS' INNATE INTELLIGENCES, MODULES, AND OPERATORS}

Why should we assess psychedelic entity experiences within the frameworks of evolutionary psychology? Simply because evolutionary psychology and the cognitive science of religion (see Atran, 2006; Barrett, 2000; Boyer, 2001, 2017; Clements, 2017; Pyysiäinen, 2009) have shown that operations of innate brain mechanisms can explain the virtual universal distribution of spirit beliefs and experiences. Of particular relevance are the perspectives on how the typical features of spirit experiences and beliefs can be explained in terms of the functions of the brain's innate operators or innate modules. The similarities of entity characteristics with the functions of humans' innate human cognitive features support the hypothesis that these innate functions are the source of entity experiences.

The operation of innate operators is revealed in the complex behaviors of newborns in a number of areas, e.g., the response of infants to faces and face-like configurations. Otsuka (2014) reviewed studies of face recognition in infants that show their selective attention to discriminate human faces. Face recognition is one of the best-studied cases of a specialized innate cognitive system (Barrett, 2000), a highly developed system which Barrett noted may reflect the need for kin recognition [Barrett (p. 176) cites research establishing the specific brain regions for facial recognition as involving "the fusiform gyrus in the inferior right temporal lobe." "Neuroimaging studies in adult participants have consistently identified cortical areas related to face processing including the inferior occipital gyrus (occipital face area), the middle fusiform gyrus (fusiform face area) and the superior temporal sulcus" (Otsuka, 2014, p. 81)]. But the system of face recognition is far more basic, exhibiting sensitivity for the overall arrangement of the separate facial features (e.g., eyes, mouth, ears, etc.), as well as evidence indicating a variety of specialized mechanisms for detecting gaze and the expression of emotions. 
The prominence of eyes in psychedelic art (i.e., Alex Grey) illustrates the prominent operation of this innate module in psychedelic experiences (also see in excess of 24 million hits for "psychedelic eye art" on Google).

Evolutionary psychology (i.e., Barkow, Leda, \& Tooby, 1992; Carruthers \& Chamberlain, 2000; Confer et al., 2010) has explanations of why we experience supernatural entities so naturally. Evolutionary psychology explains a variety of psychological phenomena as the result of biological adaptations and their behavioral, psychological, cognitive, and social functions. Evolutionary approaches to human psychology have discovered that the human mind exhibits modularity, the result of the acquisition of a separate innate modules or operators that provide specific cognitive functions (Gardner, 1983, 2000). The operation of these modules reflects capacities acquired in the course of Hominin Evolution through natural selection, particularly solutions to challenges faced by our ancestors and their hunting and gathering lifestyle.

The evolution of the human mind [Mithen (1996) has shown how the concept of innate modules explains human cognitive and social evolution. Innately disposed modular capacities are key to understanding the emergence of human cognitive uniqueness involving capacities for representation through imitation (mimesis), highly controlled physical behavior (tool use), natural history knowledge (animal behavior), social psychology (social relations and mind), and communication (music and language). Mithen proposes that it was the integration of these various modular functions that was the final cause of the major expansions in human cognitive evolution, with language serving as the mechanism through which information was exchanged among modules. Winkelman $(2002,2010)$ has shown how the effects of ritual alterations of consciousness drove these integrative processes through their manifestations in a visual modality of consciousness] involved the acquisition of specialized programs, hardwired input systems that provide for automatic information processing. These psychological adaptations respond to delimited forms of information and produce a functional output designed to provide a solution to a specific problem regularly encountered in adaptation, particularly in social relations. This evolution of specialized modular thought operators is reflected in specialized reasoning abilities in higher primates that provide cognitive functions for managing social relationships (Cummings \& Allen, 1998). Prominent among these abilities are reasoning about hierarchies and coalitions and strategies for manipulating the beliefs and behaviors of others, using a "theory of mind" to infer the motives and reasoning of others in society.

A range of findings supports a view of the human mind as manifesting a variety of unconscious functions that operate through an integrated assembly of many functionally specialized modular psychological adaptations [these adaptations are not discrete anatomical entities but are recognized because of a number of factors. These include the complexity of the behavior, the economy of function and efficiency of design, and their precision in achieving specific outcomes (Confer et al., 2010)]. The evidence that Gardner (2000) cites as establishing innate intelligences includes an explanation for their existence based on evolutionary plausibility; their role as central core cognitive operations for social life; their isolated dysfunction as a result of damage to specific brain regions; their manifestation in idiot savants and child prodigies with otherwise limited cognitive capacities; a high facility for their encoding in symbol systems; and support for their existence from experimental and psychometric studies.

Cross-cultural homologies in forms or functions of cognition involve what Laughlin, McManus, and d'Aquili (1992) call neurognostic structures, the neurobiological structures of knowing that provide the universal aspects of the human brain/mind. These neurophenomenological relations involve inherent knowledge structures of the organism that mediate the organization of experience into certain forms; these inherent structures underlie concepts such as archetypes, which are conceptualized as an ancient mode of organization of the experiences of the collective unconscious. Cross-cultural similarities in mythic accounts led the psychologist Carl Jung to propose the term archetype to represent the innate ways in which our mental hardware perceives reality as a consequence of acquired structures for representing universal aspects of human experiences. These innate dispositions of all human minds, our collective unconscious, provide the impulses that are represented in symbols and myths (summarized from Winkelman \& Baker, 2016).

Other innate structures of perception are discussed by Gardner (2000, p. 57), who identified 10 basic innate intelligences (Table 1). These capacities are inherent to the potentials of our species, but they are differentially developed as a function of individual differences, socialization influences, and environmental exposures. d'Aquili and Newberg (1999) characterize these potentials as innate systems that function not as encapsulated physical modules, but as conceptual operators linking functional components across areas of the brain that provide specific functions. They propose a number of innate modular systems that constitute the typical default processing capacities of the mind (d'Aquili \& Newberg, 1999; Table 2)

Based on Maclean's (1973, 1990) work, Ernandes (2013) discusses operators relevant to human cognition as involving a territorial operator, a hierarchic operator, a space operator, a time operator, a sequence operator, a display meaning or semiotic operator for interpretation of behavior and non-verbal communication that operates both in relationships among members of the same species as well as in interaction with different species, including the symbolic codification of behavior in primates. One of the mammalian operators involves nursing and other components of maternal care that use audiovocal communication and oxytocin release to enhance dopamine-mediated attachment dynamics to enhance maternal-offspring contact and bonding. Relevant here are both the attachment operator for establishing bonds between mother and offspring; and a "falling in love" operator that mediates the formation of coupling and pair bonding.

Ernandes proposes that some behavioral operators managed by the mammalian R-complex include "the nutrition/ homeostatic operator, for food and water needs;" "the specific operator, which allows for the acquisition of a species' identity;" "the sexual operator" and the "play 
Table 1. Gardner's $(1983,2000)$ types of innate intelligences

Original intelligences

An intrapersonal intelligence for looking in at one's own mind and the ability to use awareness of one's own capacities, desires, needs, and knowledge in achieving goals and regulating one's emotional life and relations with others.

An interpersonal intelligence, a capacity to work effectively with others through an understanding of their motivations and intentions, engaging a "theory of mind" to infer others' mental processes.

A capacity for linguistic intelligence (actually involves several capacities).

A logical-mathematical reasoning capacity for carrying out mathematical processes to solve problems, a capacity that manifests in extreme forms in the idiot savants with superhuman math processing capacities.

A bodily-kinesthetic intelligence manifested in mimesis, dancing, and the capacity to use the body to solve problems and build things.

A musical intelligence to create and perform with sound and instruments.

A spatial intelligence for creating patterns in space, ranging from navigation skills to sculpturers.

\section{Additional intelligences}

A naturalist intelligence that "demonstrates expertise in the recognition and classification of the numerous species - the flora and faunaof his or her environment" (2000, p. 36). This provides a capacity to recognize species, differentiate among species, and to identify relations between and among species.

A spiritual intelligence manifested in " $a$ desire to know about experiences and cosmic entities that are not readily apprehended in a material sense" (2000, p. 40) and providing skills in meditating, entering alterations of consciousness, and engaging with spiritual, noetic, and transcendent experiences. Within this spiritual intelligence is a personal quality that Gardner called charisma, an ability to engage in a powerful emotional contact with others that also instills in them the quest for this spiritual awareness.

An existential intelligence that reflects the cognitive aspects manifested in the spiritual intelligence, "an ability to locate oneself with respect to the furthest reaches of the cosmos, ... the significance of life, the meaning of death, .. . a concern with cosmic issues" ( $\mathrm{p} .44)$.

Table 2. Innate cognitive operators (Newberg \& d'Aquili, 2001)

A causal operator that prompts the mind to interpret experiences of the universe as a sequence of specific causes and effects, providing mechanisms of supersensible forces and powers to fulfill such explanations when direct evidence is lacking

A holistic operator that perceives a "wholeness in the midst of diversity" (p. 190), a view of reality as an integrated whole, giving humans an experience of the absolute and transcendent.

A binary operator that reduces complicated relationships to simple pairs of opposites.

A reductionist operator that allows the mind to see a whole broken down into component parts.

A quantitative operator with the capacity of abstraction of quantity from perception, engaging in operations to provide estimations of number, time, and distance.

A eureka operator that provides rapid problem-solving that reaches the consciousness suddenly through unconscious processes rather than by trial and error.

Several linguistic operators, one related to speech and based on neocortical components involving Broca's area, and one for understanding of meanings involving Wernicke's area.

operator," which provide behavioral and cognitive functions; and emotional operators, especially "fear operator" and "aggression operators," including a predatory aggression and intraspecific aggression operators.

Universal human emotions (sadness, happiness, fear, anger, disgust, and surprise) are also cases of highly specialized neural operators that make such experiences present across cultures. Damasio (1999) characterizes emotions as core biological processes based in innate brain devices that play a regulatory role through non-conscious processes that are manifested in the body. Emotions have biological roles of producing specific reactions to situations that require specific types of responses, which require action from aspects of our evolved biology that helps regulate survival behaviors. This biological machinery produces stereotyped response patterns - emotions - that are experienced as feelings and manifested in images. Consciousness emerges as the organism comes to know and experience its own emotions as information, extending the capacity to maintain homeostasis and adapt to the environment.

\section{Modular capacities in the production of supernatural experiences}

The cognitive science of religion (e.g., see Clements, 2017) has shown how the widespread human belief in the spirit world (and its virtual universality as a cultural belief) results from basic brain operators. This widespread human tendency to perceive animate entities wherever we look is a consequence of the automatic operation of modular capacities. This tendency is derived from the functions of a variety of innate human capacities (Boyer, 1992, 2001; Guthrie, 1993). Barrett (2000) proposed that supernatural belief is a direct function of a hyperactive agent-detection device (HADD). This is an automatic tendency to project the interpretation of an active agent responsible for ambiguous events. The basic function of the HADD is to attribute the intention of some agent as the cause of unexplained phenomena. This agency detection function or agency operator (Ernandes proposes that while the agency operator may acquire a rational component from the involvement of the 
neocortex's causal operator, its basis is in reptilian and limbic operators) expanded in its functions across the course of primate evolution to enhance our capacity for detection of predators. This natural selection resulted in adaptations that were overly sensitive for the detection of agency because erroneous responses (false positives) had few costs in comparison to the possible loss of life resulting from the failure to detect the presence of a predator. We are hypertuned to detect an active intentional agent where information is ambiguous or incomplete, and the perception of entities during psychedelic experiences would be a clear example of such decision-making.

This projection is facilitated by the human expansion of the assumptions of an "unseen other" with assumptions regarding the status, mental states, and intentions of these others. The human capacity for modeling others' cognitive states using our previous experiences makes it inevitable that we project our human cognitive dynamics into the interpretation of ambiguous circumstances we interpret as involving others. The unknown and ambiguous experiences within nature are animated with the projections of our own psychodynamics as the framework for the interpretation, giving our internal dynamics as the basis of our perceptions of the unknown. Thus, the ancient innate capacity for agency detection was further honed in humans for detection of human-like agents, making nature humanized (anthropomorphized) with our own self properties, social characteristics, and emotional qualities.

The experiences of spirits reflect the human capacity for a "theory of mind;" this capacity to infer the mental states and intentions of others is discussed by Gardner (2000) in terms of an innate intrapersonal intelligence. The intrapersonal intelligence is a metacognitive operator that provides an awareness of the contents of one's own mental states and the ability to relate that awareness to circumstances in the social and physical environment. This same capacity for representing our own mental states allows for the "theory of the mind," a capacity for an inferred awareness of the contents of other people's minds.

Ernandes (2013) characterizes this innate function of "theory of mind" as providing capacities for metarepresentation, a metacognitive "thinking about thinking," including thinking about the mental states of others, providing forms of "mindreading" and "mental state attribution" revealing the likely internal states of others. The ability to assess others' motives and intentions and the implications that these have for our self involves using our own self-models for representations of the mental states of those others.

Interpersonal intelligence is a social psychological operator for the internalization of the identities and properties of others. This interpersonal intelligence is a key concept for understanding a necessary aspect of human social behavior, the capacity to assume the perspectives, and the identity of social others. Collective human behavior is dependent upon processes of individual internalization of and identification with the properties of social others, especially dominant others. These processes are fundamental to human socialization and identity formation, and provide a basis for the internalization of norms and adopting for our own self the models and perspectives exhibited by socially significant others. This capacity also induces us to accept the notion of significant others with expectations for our behavior.

Spirits are typically conceptualized as being like persons with normal human minds and desires, perceive events, formulate beliefs regarding the world, and act to carry out specific intentions that are reasonable to the intuitive frameworks of humans (Winkelman, 2004). Projection of a concept of a human-like entity is an inevitable part of how humans conceptualize the unknown. We project human characteristics and an expectation of human-like entities, an inevitable aspect of default human cognitive function derived from adaptations to conditions in which we benefitted from knowing the expectations of human others and their thoughts and attitudes about us that we internalized as scripts for our self.

Our brains are further wired to detect the specific goals of the behaviors of others, embodied in the mirror neurons that fire when one performs an action - as well as when perceiving another doing the same action. This mimetic operator (Ernandes notes that the mimetic operator, localized in the amygdalar complex of the paleomammalian brain, also plays a central role in the modulation of facial expression) is an extension of a basic or communicative operator found across species, the isopraxic operator. This drives an individual to mentally represent and perform the actions observed in the behaviors of another member of the species. In humans, this isopraxic operator is extended in mimesis through the operation of mirror neurons (Garrels, 2005, 2011). Ernandes notes that a function of isopraxic operators is to produce a pattern in which animals of a group all behave in the same way. These capacities are a fundamental tool for conspecific recognition, entailing an awareness that an "other" is like one's self, a member of one's own species group.

Religious concepts of supernatural agency and causation reflect uses of a number of innate psychological systems that are linked together in ways that provided adaptive responses to the developmental and communication needs of more complex social groups. Pyysiäinen (2009, p. 13) proposed that the general dynamic of religious behavior combines the operation of the hyperactive agency detection with two other operators that he calls the "hyperactive understanding of intentionality device," "the tendency to postulate mentality and see events as intentionally caused even in the absence of a visible agent" (Pyysiäinen, 2009, p. 13) and the "hyperactive teleofunctional reasoning device," a "tendency to see objects as existing for a purpose." The innate nature of these reasoning processes is revealed in studies of children's natural tendency to offer pervasive teleological explanations of events they experience, postulating an intentional design by a supernatural agency.

Newberg and d'Aquili (2001) similarly characterize the neuropsychological mechanisms underlying religious experiences and behaviors as primarily involving a causal operator and a holistic operator. The innate function of the causal operator provides mechanisms of supersensible forces and powers to fulfill explanations with cause and effect mechanisms when direct evidence is lacking. The holistic operator perceives a wholeness to reality, an integrated whole that provides an experience of the absolute and transcendent. 
This use by religion of modular functions of the social mind provides an understanding of how supernatural thought involved exaptations of previous modules, new adaptations targeted by natural selection to enhance social behaviors through the use of natural symbols, such as animal representations of social groups manifested in totemism. Cross-cultural similarities manifested in shamanistic and mystical traditions reflect underlying innate modules which are reflected in experiences related to those modular functions, such as animal identities, soul flight, death-andrebirth, visions, and emotions of bliss, detachment, and void (Winkelman, 2010).

The operation of modules and operators in the production of religious behavior is not their independent function, but their combination to create a general model that links various elements of reality together to provide general explanatory models of how the universe operates (Ernandes, 2013). These combinations of innate intelligences are key aspects of how supernatural experiences contributed to new forms of intelligence, constituting symbols derived from the integration of operations from different cognitive modules (Winkelman, 2010). This combination of modules in the production of supernatural thought may have begun in the linking of the natural history or animal species module to the modules for personal and social identity. For instance, concepts of animacy and nature beings mix properties of personal and natural modules, while totemic groups represent the production of social identities with animal elements, exploiting modules for representing the natural world (Winkelman, 2010).

These beliefs involve the activation of the naturalist or natural history intelligence. This prehistoric huntergatherers' intuitive biology that provided a template for learning about animals was extended as a system to organize information in other domains of significance, in particular, for thinking about personal and social identity. Consequently, an innate intelligence regarding animals provided the basis for creating a universal analogical system for extension of meaning through animal metaphors as representations of personal and social identity; these provide powerful adaptations for integrating social groups (one of the most prevalent aspects of "animal" thought is found in shamanistic incorporation of animal spirits and their abilities. Typically, these animals provide a personal social relationship and aspect of personality and personal powers, or as a group symbol for spiritual and collective relationship to the group. This use of the animal world also provided a natural system of knowledge for organizing relations within a group and between societies. Totemism provides a system for differentiating societies by means of analogy, using the innate differences existing among species to represent society, representing differences among humans through the innately recognized differences among species of animals. These cognitive inventions of shamanism were a natural outcome of the integration of the different innate modules caused by ritual alterations of consciousness. These modes of experience provide access to different aspects of the self, including animal selves, as well as the disembodied self or an out-ofbody experience) (Winkelman, 2010).

\section{Hypotheses of innate functions in entity experiences}

The appearance of different innate operators in entity experiences, including the sequences of presentation and co-occurrence, should follow patterns that reflect the sequential effects of psychedelics on neurotransmitter systems, the modular operators, and the operation of the triune brain.

Hypothesis 4: The central shared properties across psychedelic and other types of entity experiences are a direct reflection of innate social modules, which will be exhibited much more than cognitive operators (i.e., mathematicallogical, language). The fact that these are perceived as entities with social significance means that the social operators are being activated.

Hypothesis 5: Psychedelics will produce entity experiences that manifest the lack of specific innate modules (i.e., absence of space, time, language abilities, and logicalmathematical reasoning). These innate capacities that do not manifest in psychedelic entity experiences should be explicable in terms of modular systems that are deactivated by psychedelics (i.e., the default mode network and the lack of sense of self).

Hypothesis 6: The features distinguishing different subtypes of psychedelic entity experiences will involve different interrelated configurations of innate operators. The hypothesis that entity experiences derive from the stimulation and release of the human modular brain functions should receive support from findings that the core distinguishing features of different subtypes of entity experiences are a reflection of the natural interactions among our innate operators (such as simultaneous experiences maternal love and suckling and infantile forms versus anger, dominance, and aggression).

Hypothesis 7: There are patterns in the sequences of operators that are manifested in the psychedelic entity experiences that reflect sequences of psychedelic action on the brain. A predictable sequence of features experienced under the influence of psychedelics would support hypotheses of entity experiences as a direct result of their brain interference. The sequences of experiences under psychedelics will reflect the order of effects on major brain and neurotransmitter systems and their consequences for the stimulation and release of these innate modules. The emergence of modular operator features in psychedelic (entity) experiences will directly reflect the sequences of return of brain functions after their destabilization by psychedelic interference with neurotransmission functions.

\section{Forms of self as innate processes}

If the alien entity experience is an internally produced experience, not an external phenomena, who is the entity that is being experienced? There are frequent reports of psychedelic entity experiences in which percipients allege being told by the entity that it is an aspect of their own self. Perhaps we should take these revelations as true. But who is this other self? 
The existence of various forms of self, with differential access to innate operators, provides a basis for different experiences of the self as an "other." There are a number of forms of self development attested to in developmental studies, psychiatric assessments, and transpersonal development models. A general perspective is that there are stages in the development of the self, with each stage superseded across time through subsequent development. These prior stages of self remain within the cognitive and emotional structures, but not normally engaged with consciousness. These other selves may however still influence behaviors and desires and figure significantly in the emotional life of the person, operating as semi-autonomous complexes, particularly in projections and dreams.

This psychologizing of psychedelic entity experiences is reflected in Gallimore and Luke's (2016) characterizations of DMT-induced experiences of dwarfs and elves as representing dynamics of the collective unconscious, particularly symbols of archetypes of the Self. They note that these can form self-contained psyches with autonomous psychic complexes potentially "capable of a consciousness of their own" (p. 12). This autonomy of alternative self forms is manifested in experiences of possession, characterized by the presence of an entity that can take control of the person's experience, voice, and body.

Process of internalization of the self of another and socially projecting it in social relations is a basic feature of the autodynamics of the human persona. This internalization and socially projection of self is manifested not only in normal experiences but also in unusual psychological phenomena where a person assumes the identity of someone else, often a spirit. This process is manifested in possession experiences, where meek, submissive, and socially marginalized women assume the personalities of dominant spirits and command a social following and high prestige for their alter status as a medium of the gods. In modern societies, these same dynamics are manifested in dissociative identity disorders (previously referred to as multiple personality disorder). These terms refer to psychological phenomena where a person has a number of different personalities and shifts between them, often in a response to trauma. Notably, most personalities have amnesia for the others, a dissociation that is also a notable feature of the compartmentalized innate modules.

Seligman and Kirmayer (2008) characterize dissociation as involving an evolved mechanism [Winkelman (2010) has addressed how dissociation is an adaptive reaction which enables an individual to continue to function in relation to their parents or caregivers by dissociating from the emotional stress that is experienced in the relationship with them. Dissociation involves a regulation of attention mechanisms that allow a selective suppression of perceptions and memories and reducing physiological stress. These processes of dissociation involve psychological defense mechanisms that allow the emotional self to continue to function in the face of trauma that has devastated the personal self. Sar and Ozturk (2007) propose that dissociation involves a detachment of the psychological self from the sociological self. This sociological self, which functions in the interface between the individual and society, allows for a reestablishment of connections between the inner and outer world (Winkelman, 2010)] that functions to relieve the effects of extreme interpersonal emotional stress. Stressful parental relationships can disrupt the integration of individual consciousness and result in the formation of a separate personality with a dissociated dynamics that results from selective suppression of memories. Dissociation provides an emotional distance from the trauma that permits an inhibition of the normal flight-or-fight response, enabling the child to seek adaptive solutions in the relationship rather than facing the risks associated with flight (Sar \& Ozturk, 2007). In the context of possession spirits, this dissociation releases mechanisms for identification with the idealized social norms that the possessing spirits present (rather than the pathological models which the parents present). In the phenomena of possession, the social roles provide mechanisms for personal control, exemplified in these higher order personalities represented in the possessing spirits who redirect the person's identity and behavior to facilitate personal and social adaptations to troubling social circumstances.

\section{Alien self phenomena}

The sense of engaging with an autonomous alien entity while in a psychedelic state has parallels with a psychiatric phenomena of alien control, where the person experiences certain contents in consciousness but they do not have a feeling that they belong to their self (Gerrans, 2015; Klein, 2015). Alien control involves experiences of one's behaviors for which one has no sense of ownership and the similar delusion of thought insertion in which people experience the presence of thoughts that they do not feel are their own (Gerrans, 2015). The delusions of alien control involve an experience of one's body performing an action without having a sense of ownership or agency for the behavior, but rather as is if the actions performed are being controlled by someone else.

Klein (2015) shows that there are a variety of recognized clinical states in which there is a lack of ownership of one's own mental states, that they are not experienced as belonging to one's self. The sense that one is not the owner of one's mental states is found associated with clinical diagnoses of schizophrenic thought insertion and somatoparaphrenia, where the person has objects in awareness, but without a feeling that they are part of one's personal consciousness. Instead, they are viewed as be alien to the self. "The feeling that this content is 'mine' is no longer present. That is, despite maintaining a clear sense of hosting a mental state (i.e., perspectival ownership), that state is not experienced as belonging to oneself" (Klein, 2015, p. 363).

These experiences can result from mental processes derived from dedicated neural networks involving subexperiential processes that may or may not be psychologically acted upon and brought into consciousness and given feelings of personal ownership. "Personal ownership is not an intrinsic property of the intentional object; rather, ownership requires that consciousness relate to its object in a particular, self-referential way" (Klein, 2015, p. 362).

Gerrans (2015) proposes a predictive coding model related to motor control as an explanation of reported 
delusions of alien control involving a loss of a sense of personal agency for the intentional actions performed by one's body. "The account of the sense of agency for imaginary action proposed here involves the following elements. The relevant intention is translated into a motor instruction that activates all the covert components of the action control system. However, because actual overt movement is inhibited, there is no translation of these instructions into movement, and the action remains covert, not overt. An important feature of the covert system is the prediction of feedback produced by attenuation of activity in the rIPL [right inferior parietal lobe]. However, because there is no proprioceptive reafference to modulate activity in the rIPL, its level of activation increases, signaling an error. Attention is captured and higher level cognitive resources are recruited, which is experienced as the sense of agency for imaginary action" (Gerrans, 2015, p. 296).

The normal sense of agency for our actions is a consequence of a proprioceptive feedback loop involving visually guided control. Gerrans notes evidence that the prefrontal brains' resolution of prediction error (involving frontalparietal circuitry) gives one the experience of being in control of an action. One's sense of agency for one's action involves processes of higher level control involved in the multisensory integration required for movement. While the actions are imagined and covert, a sense of agency emerges because of a lack of modulatory feedback activity in the rIPL. A sense of agency for these imagined actions is a consequence of the lack of reafference, which is absent because actual motor output does not occur.

The mechanism for prediction involves motor cortex signals that inhibit or attenuate the rIPL areas. Damage to the rIPL or its temporary dysfunction impairs a person's sense of agency for thought and actions they experience. Notably, the IPL is part of the default mode network (DMN) that is deactivated by psychedelics. Furthermore, hyperactivity in the right inferior parietal lobule is also associated with experiences of passive speech. Gerrans notes that when one is unable to modulate rIPL activity, the result is a loss of sense of agency for one's experiences. When excessive activity levels in predictive circuitry cannot be modulated, the subject experiences those actions as outside of personal control. In the experiences of imaginary action or imaginary speech, the motor commands that produce feedback predictions lack sensory feedback, so the predictive circuitry evoked to account for imaginary action projects an agent to account for it and attributes it to a form of alien control.

\section{Proto, core, and autobiographical selves}

The nature of the self in possession, as well as in entity experiences, may be revealed by Damasio's (1999, 2001) clinical studies that identified different forms of the self that manifest under certain conditions of compromised brain functioning. The existence of different innate forms of the self is revealed in several distinctive profiles of social, psychological, and cognitive deficiencies found in psychiatric and neurological patients. These different forms of the self include:
1. an unconscious proto-self that derives from the momentary maps of neural patterns that represent the current conditions of the physical structure of an organism;

2. a core self that depends on second-order interpretations derived from experiences of the proto-self, combined with emotions, which is experienced as a non-verbal account, a "wordless narrative" based on images that provide a causal explanation of the organism-object relationship; and

3. an extended or autobiographical self that depends on core consciousness and conventional and working memory to provide the basis for our ordinary identity and permanent sense of self.

Hypothesis 8: The qualities perceived for self and for the entity are hypothesized to manifest features of the proto-self and core self, but lacking the features of the autobiographical self. The features of psychedelic entity experiences should reflect qualities of the forms of self identified by Damasio, including the self experiences and self-related perceptions and behaviors of both the percipient's self and the entity other encountered in the psychedelic experiences. Research on the effects of psychedelics on the DMN shows that they result in the disintegration of specific forms of the self that appear to involve what Damasio calls the autobiographical self. The disintegration of this self also permits the emergence of other forms of the self.

Damasio characterizes the proto-self as lacking consciousness, without perceptions, interpretations, nor knowledge, but derived from non-conscious processes that represent the organism in the organism's own brain [Damasio (2001) proposes that the proto-self and its second-order maps are primarily produced by phylogenetically older structures associated with body regulation and representation that are along the midline of the brain in the misencephalic reticular formation (MRF), a collection of nuclei that regulate basic life processes, including wakefulness, attention, emotions, learning, and sleep]. The capacities of protoconsciousness are revealed in the continued activities of people who suffer epileptic automatisms which compromise attention, emotion, and adequate behaviors, while nonetheless enabling the person to persist with wakefulness; moving and interacting with objects; and walking through buildings without causing harm to self. Nonetheless there is no self awareness, no emotions, no response to other people, nor purposeful action. Damasio notes that "We are not conscious of the proto-self" (1999, p. 174), but that it is a point of reference that can be modified by future plans.

Damasio characterizes the core self as a consciousness as based in the organism's representations of the proto-self and derived from an account produced in response to modifications in the proto-self's auto-representations. Damasio (1999, pp. 169-170) proposes that core self and consciousness as deriving from the enhanced non-verbal image of an object and the description that results when an organism produces a representation of how it's own state of affairs has been affected by information processed regarding an object. These experiences are a non-verbal account, i.e., a "wordless narrative" based on images that 
provide a causal explanation of the organism-object relationship.

\section{DISCUSSION: MECHANISMS OF PSYCHEDELIC PRODUCTION OF ENTITY EXPERIENCES}

There is good reason to hypothesize that psychedelic entity experiences are similar phenomena to spirit encounters found in religious and spiritual traditions around the world, a reflection of a general human tendency to project humanlike qualities onto the universe. Like the psychological features of spirits and other supernatural entities, psychedelic entities manifest natural properties of the human mind that makes these experiences seem natural and compelling. These are neurophenomenological relations in the sense that the neurological activities eliciting innate response categories are responsible for the phenomenological content of experience.

Because of these innate features of our social and psychological toolkit, we are not only able to produce a cognitive and emotional world of others, but also to relate to those imagined entities who are not physically present. We easily enter into discourse with imagined social others, physical and non-physical, distant and internal, and to continue to engage with them on an ongoing basis. This involves thinking and experiencing a variety of interpersonal relations and communications with others, engaging in discourse with them, and even entering into phantasy-worlds imagining ways in which they can fulfill our needs. A central aspect of normal human thinking and the habitual and automatic operations of our socioemotional minds is the entertainment of thoughts about people who are not around, remembering what they said and imaging what they will say, making inferences about future interactions and constructing scenarios for ourselves on the basis of those experiences.

Boyer (2017) proposes that these inevitable human tendencies to construct imagined worlds populated with human-like entities is the consequence of our great dependence on social relations and the potential costs of mismanagement of them. Humans have evolved an intuitive theory of mind and an over-developed social intelligence because of the importance of knowing what other members of our group as well as other groups - are thinking and intending to do. "Compared to other species, humans can use vastly more complex computational tools to infer from observed behavior [others'] mental states, perceptions, beliefs, and desires" (Boyer, 2017, p. 23). Imagining possible scenarios of future interactions and possible reactions is a preparation for timely responses when situations arise.

Scenario construction is an adaptation for excelling in conditions of hypersociability and complex social interactions, a preparation for possible occurrences. Imagination of possible future responses is a preparation for performing them. "Fiction serves a functional role in human cognition, providing us with harmless, simulated situations in which to hone our social skills. Fiction functions similarly to the evolutionary role of play" (Boyer, 2017, p. 22). We appear to have an innate tendency to create models of future scenarios into which we project ourselves, and much of it may be more of a wish for phantasy than a likely scenario. A variety of products of the human imagination such as daydreaming, active imagination, fantasies, and dreams and our capacity to produce fiction all reflect common underlying innate capacities of the human mind. This scenario constructing processes, combined with our intrapersonal and interpersonal intelligences, provides the basis for content of psychedelic entity experiences and spiritual beliefs and experiences.

\section{The phantasy mode of consciousness}

Our capacity to experience interactions with human-like entities - spirit or psychedelic - must be placed in the context of our broader capacity for engaging in phantasy. Horváth, Szummer, and Szabo (2017) propose that psychedelic visionary phenomena reflect the activation of a modular-like representational system for phantasy that blends various cognitive processes. Lohmar (2016, p. 20; also see Lohmar, 2010) proposes that this phantasy system involves a form of expressive thinking that predates language, functioning as a non-linguistic method for solving important tasks. This form of thought involves the use of image schemas which represent basic structures of sensorimotor experience. This expressive system is nonetheless symbolic. It has a significant role in providing a medium for three forms of support that are necessary for thinking. Lohmar (2016, paraphrase) notes that visionary phantasy provides the ability to retain in mind an object; engender other cognitions regarding this image object; and manipulate these to consider future possibilities. Lohmar argues that this form of thinking evolved to facilitate decision-making in situations with very mixed motives. The phantasy mode of consciousness involves a range of conscious acts and mentations that blend into the processes of perceiving, anticipating events, and planning for the future, as well as engaging memories and fantasies. Visionary phantasy is intimately related to a personal representational capacity that directly reveals to the person an imaginative and familiar presentation of affective layers of consciousness.

Horváth et al. (2017) suggest that this phantasy capacity functions constantly in our day-to-day activities, providing a synthesis of perception, affect, and thinking. In the case of alterations of consciousness, it often extremely produces exotic forms of experience with their own story-line narratives and revelations. When released by psychedelics, this latent human cognitive visionary capacity takes control of consciousness, providing an internal engagement with a level of the mind that presents a narrative regarding some significant aspects of the affective dynamics of the person.

Phantasy-consciousness emerges from reworking these experiences into more exotic forms of consciousness through embellishment with story-line narratives that contain personally significant revelations. This visionary phantasy is intimately related to innate representational capacities that directly reveal to subject an imaginative presentation of material from their own personal unconscious. The psychedelic visionary state typifies phantasy experiences, a polysemic manifestation involving intense affective responses, visual experiences and imagination, as well as significant 
intellectual realizations and personal awareness (also see Winkelman, 2010 on presentational symbolism).

\section{Neurophenomenological mechanisms underlying psychedelic experiences}

To date, we have no compelling reasons to consider psychedelic entity experiences as basically any different than guardian spirits, hauntings, elf visitations, or any number of other experiences of spirits. Furthermore, we have good reason to explain the worldwide distribution of beliefs in supernatural entities to the operation of innate brain functions. Evidence that psychedelic entity experiences reflect manifestations of such innate modular brain functions as the mimetic operator is also supported by evidence derived from the psychedelic effects on brain functions that provide plausible mechanisms for psychedelic visionary experiences (Winkelman, 2017).

But why should psychedelics so powerfully elicit these kinds of experiences of the alien other, whether in the shamanic or modern context? The answer must be sought in the approaches of neurophenomenology that examine the relationship between brain operation and experience. Neurophenomenology examines how the structure and content of phenomenal experience can be related to functions at the neurological level (Laughlin et al., 1992).

As an example, an apparent neurophenomenological effect of psychedelics involves what Kent (2010) referred to as frame stacking, a repetition of edges that is reflected in the appearance of objects. These phenomena of repeated edges are a consequence of the breakdown of screening and information integration processes normally imposed by the serotonergic system in its regulation of lower brain structures. The resistance of psychedelics to the usual serotonin reuptake mechanisms causes a repeated firing of a neuron, rather than allowing its deactivation through reuptake mechanisms; this repeated firing is presumably responsible for this visual phenomena manifested in psychedelic experiences and art around the world.

In the case of psychedelic entity experiences, neurophenomenology is concerned with how psychedelic effects on brain processes produce the effects of experiencing spirits. Why should psychedelics stimulate the release of these innate modular operations of the brain?

\section{Deregulation of frontal brain structures}

Common features of spirit entity experiences must be assessed in relationship to known cognitive effects resulting from the alteration of consciousness on the brain's perceptual mechanisms and representational capacities. There are similarities in the effects of diverse conditions that alter consciousness (i.e., endurance running, hypnosis and meditation, as well as drug-induced states and dreaming) that involve a temporary deregulation of the prefrontal cortex (PFC; Dietrich, 2003). Common effects of this disruption are manifested in the loss of the roles of the frontal lobes and PFC involving higher cognitive functions. This disruption of the PFC results in the loss of various capacities - capacity for willful action, deliberate direction of attention, and aspects of self-awareness, as well as the capacities for abstract thought, creativity, and planning. When these higher level brain functions of the cortical regions and the PFC are downregulated, this allows for the manifestation of lower brain structures usually repressed by the PFC. This means the emergence of information and aspects of identity that are related to our more ancient brain regions.

A basic neurophenomenological dynamic of psychedelic experiences are revealed in studies (Carhart-Harris et al., 2012, 2014, 2016) on their effects on a brain region known as DMN, a key region for the integration of information about self and others. There were strong correlation of scores on experiences of "ego-dissolution" with decreases in connectivity between specific areas of the DMN (CarhartHarris et al., 2016; Lebedev et al., 2015), suggesting that a number of features of psychedelic entity experiences can be explained by virtue of such neurological effects. Destabilization of the DMN and ego dissolution likely explains many of the alien other effects produced by psychedelics, allowing unconscious aspects of the self to be experienced as an alien.

Psychedelic disruption of the DMN permits the operation of a more fluid and dynamic brain lacking its usual topdown principles of control. Psychedelics such as LSD, psilocybin, and ayahuasca cause decreases in DMN brain activity (Carhart-Harris et al., 2012, 2014, 2016; PalhanoFontes et al., 2015) and the disintegration of normal DMN functions. This is the consequence of a reduction in the connectivity of the frontal cortex with lower brain areas (Alonso, Romero, Mañanas, \& Riba, 2015) and the reduction in oscillatory activity and power in posterior and frontal association cortices (Muthukumaraswamy et al., 2013). This involves a decrease in the functional coupling of the frontal cortex with the medial temporal lobe, as well as of the medial PFC with the posterior cingulate cortex. Consequently, the lower brain dynamics involving ascending circuitry are released, providing a strong input to the frontal cortex from the ancient brain systems. This dynamic is hypothesized as the mechanism that releases the innate modules and promotes their manifestations in consciousness (also see Winkelman, 2017).

\section{Bottom-up information dynamics}

A principal effect of psychedelics involves production of hypersynchronous ascending slow-wave brain discharges in the hippocampal-septal-reticular-raphe circuit that impose impulses from the ancient lower stratum of the brain on the frontal areas (Mandell, 1980). This pattern of psychedelic action on the brain is shared by many other agents and conditions that alter consciousness (Winkelman, 2011). Alterations of consciousness produced by behavioral and physiological conditions, as well as pharmacological agents, cause a reduction in the serotonin inhibition to the hippocampal cells, which results in an increase in slow-wave EEG activity in the hippocampal-septal region.

Research on the mechanisms of action of psychedelics on the major cortical loops (Vollenweider \& Geyer, 2001) illustrates these effects. Psychedelic effects on the corticostriato-thalamo-cortical (CSTC) feedback loops and their regulatory effects on the gating systems of lower levels of the brain alter consciousness. This psychedelic interruption of the CSTC loops and their inhibitory functions release 
the lower brain structures' sensory gating and enhance the flow of information to the frontal areas of the brain. Psychedelic interruption of thalamic screening results in a flood of information from these ancient levels of the brain.

Alonso et al. (2015) confirmed this psychedelic enhancement of a bottom-up information dynamics by psychedelics with ayahuasca. They assessed ayahuasca-induced changes in directionality of information flow in the brain, with changes in the connectivity of brain oscillations. These changes involved a disruption of the normal coupling between anterior and posterior areas of the brain that resulted from reduction in the influence of frontal brain areas over the posterior areas. This reduction was accompanied by increases in the influence of posterior brain regions on the frontal anterior areas. "These results suggest that psychedelics induce a temporary disruption of neural hierarchies by reducing top-down control and increasing bottom-up information transfer in the human brain" (Alonso et al., 2015, p. 1).

The release of these lower brain areas is the likely cause of the enhanced operation of the innate modular operators of the brain. These operators reflect unconscious cognitive processes that were acquired deep in evolution, with some apparently widely shared by other primates and mammals. These ancient roots of these operators suggest that they are associated with the function of our ancient brain structures. Winkelman (2010) has detailed how many features of shamanic alterations of consciousness can be explained with reference to the operation of these ancient brain structures, particularly the paleomammalian brain.

\section{The paleomammalian brain in psychedelic experience}

The operations of the paleomammalian brain are also directly relevant to explaining what Gallimore and Luke (2016) note as a powerful shock of the DMT experience caused by an unshakeable feeling of authenticity that makes it impossible for the individual to deny the reality of the experience, nor dismiss it as an hallucination, in spite of its bizarre nature. For many, there is an absolute certainty regarding the reality of the DMT experience that clashes so powerfully with people's most basic assumptions regarding reality that it produces a state of "ontological shock" (Mack, 1999) regarding the ultimate nature of reality. Perhaps a totally different reality is what we would expect if most of our ordinary innate operators are disabled, linked in unaccustomed ways, or are not integrated with the self because of the destabilization of the DMN.

One mammalian operator with specific relevance to the explanation of ontological shock of psychedelic entity experiences is the "existential (or ontological) operator [which] assigns a sense of existence or non-existence to the sensory information processed by the brain... [T]his operator gives a sense of reality to beliefs, regardless of whether they are non-contradictory or contradictory, or counterintuitive, according to neocortical operators... In humans, the existential operator, which is cognitive but not necessarily rational, is linked to limbic emotional operators" (Ernandes, p. 33). This operator is fundamental to the strong feelings that the experiences reflect fundamental truths about the universe and the associated conviction regarding their reality that frequently accompanies psychedelic experiences. The firm convictions produced by psychedelics not only regarding the reality of the psychedelic entities, but of other revelations as well, implicate the operation of these paleomammalian brain circuits. As MacLean noted (1973, p. 123), "It seems that the ancient limbic system provides the ingredients for the strong affective feeling or conviction that we attach to our beliefs, regardless of whether they are true or false!"

We can further attribute the ontological certainty of the psychedelic entity experiences to the consequences of the extensive stimulation by psychedelics of our basic innate programs for organizing experience of reality. In essence, psychedelic effects in eliciting the innate modules and operators reflect an overstimulation of these systems, a supercharged activation of our categories for representing reality. This neurological effect is likely a consequence of the resistance of the 5-HT2 psychedelics to normal 5-HT2 reuptake, leaving the psychedelic molecule embedded in the receptor site where it stimulates the receptor to the point of habituation and deactivation. There are also extensive effects of DMT on the receptor systems in stimulating innate cognitive organs.

\section{Psychedelic effects on receptors and mental organs explain ontological certainty regarding DMT entities}

The reason that psychedelics in general, and DMT in particular, produce an "ontological shock," a certainty of a more compelling experience of reality than ordinary reality, is revealed by Ray's (2010) findings. Ray challenges the predominant theory of psychedelic action being mediated primarily by action at the serotonin- 2 receptors (especially 5-HT2A). Ray's (2010) study of the interaction of 25 psychedelics at 51 receptors, transporters, and ion channels found an affinity of the psychedelic drugs for 18 different sites. The historical model of psychedelic drug action primarily through effects on serotonin receptors contrasts with the dramatic differences in subjective reports from different chemical classes of substances (see Shulgin \& Shulgin, 1991, 1997). Ray (2010, 2012, 2016) shows that diverse psychedelic drug profiles reflect their interaction with dozens of different receptors, with different subjective effects associated with each psychedelic substance being the result of the diversity of interactions of various psychedelics with different receptor systems.

Ray (2012) proposes that this diverse phenomenology of psychedelic experience reflects their elicitation of the operation of different mental organs and the capacities that are the result of the expression of specific genes that underlie the evolution of specific aspects of the mind. Mental organs involve a population of neurons that have common properties and provide a specific mental function through the expression of a specific modulatory receptor (e.g., 5-HT1, 5-HT2, 5-HT7, alpha-1, alpha-2, beta, dopamine, histamine-1, imidazoline-1, kappa, mu, sigma, and cannabinoid-1). The mental organs are defined in terms of the neurotransmitter receptor that they express, thereby reflecting the structure of the brain and manifesting the structure and content of the mind's functions. Ray considers the concept of mental organs to be synonymous with 
receptor in many instances, with receptor representing molecular dynamics and mental organ reflecting the mental level.

Ray characterizes these mental organs as ordinarily unconscious, but through receptor stimulation enter into consciousness to provide a system for describing the contents of the mind, serving to represent internal, external, and social aspects of reality, as well as functions of language, logic, and reason, among others. The diverse mental organs revealed in the typical and distinct effects of various psychedelics have the functions of providing different forms of consciousness (i.e., adult vs. childhood); different time scales (long term vs. short term): salience to specific kinds of contents of consciousness; ontological categories; aspects of religious (through soul, compassion, and forgiveness); and specific mental functions (i.e., logic and language). Ray notes the frequency with which mental organs have an affective quality, giving consciousness a sense of feeling through which humans experience consciousness. Their effects on consciousness are not normally individual, but rather combined together to give a global perception of reality.

Ray proposes that the actions of psychedelic drugs on consciousness involve two major dimensions, breadth and depth, involving respectively: the number of different mental organs that are activated by a substance (breadth) and the intensity of consciousness (depth). Ray considers 5-HT2 and 5-HT7 to play intimately intertwined roles in that 5-HT2 shapes the breadth of consciousness by providing the content through integrating various mental organs, and 5-HT7 mediates the depth of consciousness. Breadth increases as a function of the number of mental organs that provide input to consciousness, expanding the contents of consciousness, with increases in the number of mental organs in consciousness producing a more complex and multifaceted representation of reality. Increasing depth is a function of increasing activation of 5-HT7 that provides "resolution, subtlety, nuance, complexity, tangibility, vividness, and capacity; to render thought, feelings, and sensory input' (Ray, n.d., p. 2). The increase in depth is responsible for the ability of psychedelic drugs to have dramatic effects such as visual experiences and ego-loss and at extremely high levels, producing a discontinuity in the contents of consciousness and a loss of contact with ordinary reality during which the organs of consciousness become more salient than external reality.

Of the psychedelic drugs that Ray assayed, DMT had the greatest depth of effect (relative affinity) and the greatest breadth of action (see Ray, 2010, Table 1, p. 19) with the receptorome, the total set of receptor proteins or the genes that encodes for traditional receptors, receptor molecules, as well as ion channels and transporters.

This combination of greatest breadth of mental organs (receptors) and greatest depth of consciousness attests to the special relations of DMT to the human neurotransmitter system that enables DMT to produce experiences of reality that are more profound and multifaceted than our ordinary experiences of reality. The DMT-mediated expansion of depth of consciousness through effects on 5-HT7 is what stimulates the creative genius and imagination beyond the experiences of actual reality. The experience of intense and complicated visionary experiences is a consequence of the great breadth of interaction that DMT has with the receptorome and consequently, the stimulation of a fuller range of mental organs. In addition, DMT is considered to be one of the most visual of all psychedelics, a property that enables it to produce a compelling experience of an alternate reality that is able to challenge one's ordinary concepts of reality.

Ray also found that DMT was psychedelic with the highest relative affinity for any dopamine receptor, with the highest relative affinity for D1. Ray characterizes dopamine as an atypical mental organ in that rather than representing some cognitive capacity or aspect of reality, dopamine gives salience to conscious experience, marking both cognitive and affective content with significance for our biographical memory, imprinting ideas, memories, and social ideologies at deep levels. Dopamine has the ability to produce feelings of awe, combined with an uncritical sense of certainty to experiences, especially those involving religious and spiritual sentiment. The activation of dopamine by psychedelic drugs in general, and especially the very strong action at dopamine receptors by DMT, induces the capacity to allow new social constructs to take precedence over past learning and produce extreme changes in beliefs and behaviors.

In assessing the affinity of DMT across 17 types of receptor sites (5-HT7, 5-HT1D, 5-HT2B, alpha2B, alpha2C, dopamine1, 5-HT2C, 5-HT1E, 5-HT6, 5-HT5A, imidazoline1, alpha1B, alpha2A, alpha1A, 5-HT2A, SERT, and sigma1) and in contrast with 28 other psychoactive drugs (mostly psychedelics), Ray (2010) found that affinity profile of DMT had a number of distinguishing properties in addition to its exceptionally high affinity for all dopamine receptors. The relative affinity (normalized) of DMT at 5-HT7 was the highest affinity of any of the 29 drugs assayed; DMT also had the highest relative affinity at 5-HT1D, 5-HT1E, D1, alpha1A, alpha1B, and alpha2B receptor sites. DMT also had the second greatest relative affinity at 5-HT2C, the third greatest relative affinity at 5-HT5A and alpha2C, and the fourth greatest relative affinity at 5-HT6 and sigma1. Ray concludes that DMT is the neurotransmitter with the greatest breadth of interaction across all receptor sites, the second greatest breadth of interaction across 10 serotonin receptors, 7 adrenergic receptors, and the 3 alpha 2 receptors assayed. This exceptionally high relative affinity of DMT for many receptor sites contrasts with the relatively little affinity of serotonin, dopamine, and norepinephrine for each other's receptors.

"This is a long list of exceptional properties for any one drug. DMT is truly distinctive" (Thomas Ray, personal communication). The ability to stimulate so many receptor systems at such high levels offers a plausible explanation as to why the DMT-induced entity experiences give such a powerful sense of ontological certainty. This ability of DMT to construct a convincing alternate reality illustrated in the DMT entities and their ontological certainty is a reflection of the many receptors and mental organs that DMT stimulates and forces into consciousness. The ability to stimulate many different mental organs, far more than typical of ordinary consciousness, is why DMT experiences are considered to reveal a credible reality that is perceived as more real than ordinary reality (which is constructed by the integration of fewer mental organs). Furthermore, because of the large number of non-serotonin mental organs activated by DMT, it produces an experience that is more multifaceted than ordinary reality with rich, realistic details that provide a convincing experience of an alternate reality. 


\section{CONCLUSIONS}

We need to assess psychedelic entity experiences with respect to known projective properties of the human mind and its creative spirit, and specifically with respect to the innate modules that have been useful in explaining the prevalence of spirit experiences and beliefs. Our innate modules give us programs that worry about what others think of us and imaging what they must be thinking and planning to do. Comparisons of entity experiences with the great creative works of fiction literature, movies, and the manifestations of epic dreams suggest that psychedelic entities experiences do not substantially exceed anything experienced in dreams, novels, films, or other expressive productions, including our famed myth-making and story-telling capabilities. Humans believe in their religious myths as an unshakable ultimate reality, so it should not be considered surprising or unusual that many also have unshakable beliefs in the visionary experiences induced by psychedelics.

If humans can do unconsciously and through automatic and unconscious innate modules anything that a psychedelic entity does, what is left to explain but an active imagination externalized in a psychedelic experience? Without evidence of psychedelic entity behavior that manifests a complexity of interaction that exceeds the ordinary default dynamics of human interaction, we have nothing to explain. Unless someone can show that psychedelic entity experiences exceed such productions of the human consciousness, or that they can provide information under conditions of control exemplified in parapsychological experiments, I think that we are forced to interpret psychedelic entity experiences as reflecting projective capacities of the human mind.

Situating psychedelic entity experiences within a broad range of similar entity experiences may provide evidence of noumena behind these diverse but similar phenomenal manifestations. But evidence of the existence of a noumenon does not establish the ontological reality of the claims made about it. The conceptual explanation of entities provided here proposes that their properties can be explained in terms of the overstimulation of ordinary innate cognitive processes.

But even if psychedelic entity experiences are basically similar to other entity experiences, and furthermore reflect the basic categories of our innate modular psychology, psychedelics nonetheless remain as an important tool for exploring the conditions of these experiences of entities. Humans' innate psychology may - or may not - explain all of these experiences, but in any case, psychedelics will remain a key tool for the exploration of the general brain conditions facilitating these types of experiences.

Funding sources: The author had no funding for this article.

Author's contribution: MJW and his psychedelic entities are responsible for all aspects of this article.

Conflict of interest: The author has no conflict of interest to declare.
Ethics: The author's research raised no ethical concerns, being exclusively based on published sources.

Acknowledgements: The author would like to thank Nicholas Sampson for helpful suggestions in formulating this paper and Heather Hargraves for comments on a draft of the paper.

\section{REFERENCES}

Alonso, J. F., Romero, S., Mañanas, M., \& Riba, J. (2015). Serotonergic psychedelics temporarily modify information transfer in humans. International Journal of Neuropsychopharmacology, 18(8), 1-9. doi:10.1093/ijnp/pyv039

Atran, S. (2006). The cognitive and evolutionary roots of religion. In P. McNamara (Ed.), Where God and science meet: How brain and evolutionary studies alter our understanding of religion Vol 1: Evolution, genes, and the religious brain (pp. 181-207). Westport, CT: Praeger.

Barkow, J., Leda, C., \& Tooby, J. (Eds.). (1992). The adapted mind: Evolutionary psychology and the generation of culture. New York, NY: Oxford University.

Barrett, J. (2000). Exploring the natural foundations of religion. Trends in Cognitive Sciences, 4(1), 29-34. doi:10.1016/S13646613(99)01419-9

Boyer, P. (1992). The naturalness of religious ideas. Berkeley, CA: University of California Press.

Boyer, P. (2001). Religion explained: The evolutionary origins of religious thought. New York, NY: Basic Books.

Boyer, P. (2017). Imagining superhuman agents. In N. Clements (Ed.) Macmillan interdisciplinary handbooks. Religion. Mental religion (pp. 17-30). Farmington Hills, MI: Macmillan Reference.

Carhart-Harris, R. L., Errotzoe, D., Williams, T., Stone, J. M., Reed, L. J., Colasantia, A., Tyackea, R. J., Leechd, R., Maliziab, A. L., Murphye, K., Hobdene, P., Evanse, J., Feilding, A., Wisee, R. G., \& Nutta, D. J. (2012). Neural correlates of the psychedelic state as determined by fMRI studies with psilocybin. Proceedings of the National Academy of Sciences of the United States of America, 109(6), 21382143. doi:10.1073/pnas.1119598109

Carhart-Harris, R. L., Leech, R., Hellyer, P. J., Shanahan, M., Feilding, A., Tagliazucchi, E., Chialvo, D. R., \& Nutt, D. (2014). The entropic brain: A theory of conscious states informed by neuroimaging research with psychedelic drugs. Frontiers in Human Neuroscience, 8, 20. doi:10.3389/ fnhum.2014.00020

Carhart-Harris, R. L., Muthukumaraswamy, S., Roseman, L., Kaelen, M., Droog, W., Murphy, K., Tagliazucchi, E., Schenberg, E. E., Nest, T., Orban, C., Leech, R., Williams, L. T., Williams, T. M., Bolstridge, M., Sessa, B., McGonigle, J., Sereno, M. I., Nichols, D., Hellyer, P. J., Hobden, P., Evans, J., Singh, K. D., Wise, R. G., Curran, H. V., Feilding, A., \& Nutt, D. J. (2016). Neural correlates of the LSD experience revealed by multimodal neuroimaging. Proceedings of the National Academic Science of the United States of America, 113(17), 4853-4858. doi:10.1073/pnas.1518377113

Carr, S. (1995). Exquisitely simple or incredibly complex: The theory of entoptic phenomena. Retrieved from http://www. oubliette.org.uk/Intro.html 
Carruthers, P., \& Chamberlain, A., (Eds.). (2000). Evolution and the human mind: Modularity, language, and meta-cognition. Cambridge, UK: Cambridge University Press.

Clements, N. (Ed.). (2017). Macmillan interdisciplinary handbooks. Religion. Mental Religion. Farmington Hills, MI: Macmillan Reference.

Confer, J., Easton, D., Fleischman, C., Goetz, D., Lewis, G., Perilloux, C., \& Buss, D. M. (2010). Evolutionary psychology controversy questions, prospects and limitations. American Psychologist, 65(2), 110-126. doi:10.1037/ a0018413

Cummings, D., \& Allen, C., (Eds.). (1998). The evolution of mind. Oxford, UK: Oxford University Press.

Damasio, A. (1999). The feeling of what happens. Orlando, FL: Harvest Book/Harcourt.

Damasio, A. (2001). Emotion and the human brain. Annals of the New York Academy of Sciences, 935(1), 101-106. doi:10.1111/ j.1749-6632.2001.tb03475.x

d'Aquili, E., \& Newberg, A. (1999). The mystical mind: Probing the biology of religious experience. Minneapolis, MN: Fortress Press.

Dietrich, A. (2003). Functional neuroanatomy of altered states of consciousness: The transient hypofrontality hypothesis. Consciousness and Cognition, 12(2), 231-256. doi:10.1016/ S1053-8100(02)00046-6

Dittrich, A. (1998). The standardized psychometric assessment of altered states of consciousness (ASCs) in humans. Pharmacopsychiatry, 31(Suppl. 2), 80-84. doi:10.1055/s2007-979351

Dittrich, A., Von Arx, S., \& Staub, S. (1981). International study on altered states of consciousness (ISASC). Schweizerische Zeitschrift für Psychologie und ihre Anwendungen/Revue suisse de Psychologie pure et appliquée, 40(3), 189-200.

Dittrich, A., Von Arx, S., \& Staub, S. (1985). International study on altered states of consciousness (ISASC). Summary of the results. German Journal of Psychology, 9, 319-339.

Ernandes, M. (2013). The triune brain and the neurobiological bases of thought and behaviour. In R. Cuoghi \& M. Farronato (Eds.), De Incontinentia (pp. 52-87). Bologna, Italy: Mouse Publishing.

Fox, M., \& Sheldrake, R. (2014). The physics of angels: Exploring the realm where science and spirit meet. New York, NY: Monkfish Books Publishing.

Gallimore, A., \& Luke, D. (2016). DMT research from 1956 to the edge of time. In D. King, D. Luke, B. Sessa, C. Adams, \& A. Tollen (Eds.), Neurotransmissions essays on psychedelics from breaking convention (pp. 291-316). London: Strange Attractor Press.

Gardner, H. E. (1983). Frames of mind: The theory of multiple intelligences. New York, NY: Basic Books.

Gardner, H. E. (2000). Intelligence reframed: Multiple intelligences for the 21st century. New York, NY: Basic Books.

Garrels, S. (2005). Imitation, mirror neurons, and mimetic culture: Convergence between the mimetic theory of Rene Girard and empirical research on imitation. Contagion: Journal of Mimesis, Violence and Culture, 12(1), 47-86. doi:10.1353/ ctn.0.0004

Garrels, S. R. (Ed.). (2011). Mimesis and science empirical research on imitation and the mimetic theory of culture and religion. East Lansing, MI: University of Michigan Press.
Gerrans, P. (2015). The feeling of thinking: Sense of agency in delusions of thought insertion. Psychology of Consciousness: Theory, Research, and Practice, 2(3), 291-300. doi:10.1037/ cns0000060

Guthrie, S. (1993). Faces in the clouds: A new theory of religion. Oxford, UK: Oxford University Press.

Hancock, G. (2000). Supernatural: Meeting with the ancient teachers of mankind. London: Century.

Horváth, L., Szummer, C., \& Szabo, A. (2017). Weak phantasy and visionary phantasy: The phenomenological significance of altered states of consciousness. Phenomenology and the Cognitive Sciences, 17(1), 117-129. doi:10.1007/s11097016-9497-4

Kenny, A. (2012). A new history of Western Philosophy. Oxford, UK: Oxford University Press.

Kent, J. L. (2010). Psychedelic information theory: Shamanism in the age of reason. Seattle, WA: PIT Press.

Klein, S. B. (2015). The feeling of personal ownership of one's mental states: A conceptual argument and empirical evidence for an essential, but underappreciated, mechanism of mind. Psychology of Consciousness: Theory, Research, and Practice, 2(4), 355-376. doi:10.1037/cns0000052

Kluver, H. (1928). Mescal - The divine plant and its psychological effects. London, UK: Kegan Paul, Trench, Trubner \& Co.

Laughlin, C., McManus, J., \& d'Aquili, E. (1992). Brain, symbol, and experience toward a neurophenomenology of consciousness. New York, NY: Columbia University Press.

Lebedev, A., Lövdén, M., Rosenthal, G., Feilding, A., Nutt, D., \& Carhart-Harris, R. (2015). Finding the self by losing the self: Neural correlates of ego-dissolution under psilocybin. Human Brain Mapping, 36(8), 3137-3153. doi:10.1002/hbm.22833

Lohmar, D. (2010). The function of weak phantasy in perception and thinking. In S. Gallagher, \& D. Schmicking (Eds.), Handbook of phenomenology and cognitive science (pp. 159-177). London: Springer.

Lohmar, D. (2016). Emotions as a multi-modal system of representation in non-linguistic thinking. Phenomenology and Mind, 11, 20-30. doi:10.13128/Phe_Mi-20103

Luke, D. (2011). Discarnate entities and dimethyltryptamine (DMT): Psychopharmacology, phenomenology and ontology. Journal of the Society for Psychical Research, 75(902), 26-42.

Luna, L., \& Amaringo, P. (1999). Ayahuasca visions: The religious iconography of a Peruvian shaman. Berkeley, CA: North Atlantic Books.

Mack, J. E. (1999). Passport to the cosmos: Human transformation and alien encounters. New York, NY: Three Rivers.

MacLean, P. (1973). The triune concept of brain and behavior. Toronto, ON: University of Toronto Press.

MacLean, P. (1990). The triune brain in evolution. New York, NY: Plenum Press.

Mandell, A. (1980). Toward a psychobiology of transcendence: God in the brain. In D. Davidson \& R. Davidson (Eds.), The psychobiology of consciousness (pp. 379-464). New York, NY: Plenum.

Merriam-Webster Dictionary. (2017). Entity. Springfield, MA. Retrieved from https://www.merriam-webster.com/

Meyer, P. (1994). Apparent communication with discarnate entities induced by dimethyltryptamine. In T. Lyttle (Ed.), Psychedelics (pp. 61-203). New York, NY: Barricade Books.

Meyer, P. (2010). 340 DMT trip reports. Retrieved from www. serendipity.li/dmt/340_dmt_trip_reports.htm 
Mithen, S. (1996). The prehistory of the mind: A search for the origins of art, religion, and science. London: Thames and Hudson.

Muthukumaraswamy, S. D., Carhart-Harris, R. L., Moran, R. J., Brookes, M. J., Williams, T. M., Errtizoe, D., Sessa, B., Papadopoulos, A., Bolstridge, M., Singh, K. D., Feilding, A., Friston, K. J., \& Nutt, D. J. (2013). Broadband cortical desynchronization underlies the human psychedelic state. Journal of Neuroscience, 33, 15171-15183. doi:10.1523/ JNEUROSCI.2063-13.2013

Newberg, A., \& d'Aquili, E. (2001). Why God won't go away. Brain science and the biology of belief. New York, NY: Ballantine Books.

Otsuka, Y. (2014). Face recognition in infants: A review of behavioral and near-infrared spectroscopic studies. Japanese Psychological Research, 56(1), 76-90. doi:10.1111/jpr.12024

Palhano-Fontes, F., Andrade, K. C., Tofoli, L. F., Santos, A. C., Crippa, J. A. S., Hallak, J. E. C., Ribeiro, S., \& de Araujo, D. B. (2015). The psychedelic state induced by ayahuasca modulates the activity and connectivity of the default mode network. PLoS One, 10(2), e0118143. doi:10.1371/journal.pone.0118143

Pekala, R., Steinberg, J., \& Kumar, V. (1986). Measurement of phenomenological experience: Phenomenology of consciousness inventory. Perceptual and Motor Skills, 63(2), 983-989. doi:10.2466/pms.1986.63.2.983

Pyysiäinen, I. (2009). Supernatural agents. Why we believe in souls, gods, and buddhas. London/New York: Oxford University Press.

Ray, T. (2010). Psychedelics and the human receptorome. PLoS One, 5(3), 1-41. doi:10.1371/annotation/e580a864-cf13-40c29bd9-b9687a6f0fe4

Ray, T. (2012). Mental organs and the origins of mind. In L. Swan (Ed.), Origins of mind (pp. 301-326). New York/Heidelberg: Springer.

Ray, T. (2016). Constructing the ecstasy of MDMA from its component mental organs: Proposing the primer/probe method. Medical Hypotheses, 87, 48-60. doi:10.1016/j.mehy.2015.12.018

Ray, T. (n.d.). Mental organs and the breadth \& depth of consciousness. Retrieved from https://www.transformpress. com/breadth-and-depth

Sar, V., \& Ozturk, E. (2007). Functional dissociation of the self: A sociocognitive approach to trauma and dissociation. Journal of Trauma and Dissociation, 8(4), 69-89. doi:10.1300/ J229v08n04_05

Seligman, R., \& Kirmayer, L. (2008). Dissociative experience and cultural neuroscience: Narrative, metaphor and mechanism. Medicine \& Psychiatry, 32(1), 31-64. doi:10.1007/s11013007-9077-8

Shanon, B. (2010). The epistemics of ayahuasca visions. Phenomenology and the Cognition Science, 9, 263-280. doi:10.1007/ s11097-010-9161-3
Shulgin, A., \& Shulgin, A. (1991). PIHKAL: A chemical love story. Berkeley, CA: Transform Press.

Shulgin, A., \& Shulgin, A. (1997). TIHKAL: The continuation. Berkeley, CA: Transform Press.

Sidgwick, H., Johnson, A., Myers, F. W. H., Podmore, F., \& Sidgwick, E. M. (1894). Report on the census of hallucinations. Proceedings of the Society for Psychical Research, 10, 25-422.

Siegel, R. K. (1977). Hallucinations. Scientific American, 237(4), 132-139. doi:10.1038/scientificamerican1077-132

St John, G. (2015). Mystery school in hyperspace: A cultural history of DMT. New York, NY: Evolver Editions.

Strassman, R. (2000). DMT: The spirit molecule: A doctor's revolutionary research into the biology of near-death and mystical experiences. Rochester, VT: Inner Traditions.

Studerus, E, Gamma, A., \& Vollenweider, F. (2010). Psychometric evaluation of the altered states of consciousness rating scale (OAV). PLoS One, 5(8), e12412. doi:10.1371/journal.pone. 0012412

The Encyclopedia of Philosophy. (2017). Logic and ontology. Retrieved from https://plato.stanford.edu/entries/logic-ontology/

Vollenweider, F., \& Geyer, M. (2001). A systems model of altered consciousness: Integrating natural and drug psychoses. Brain Research Bulletin, 56(5), 495-507. doi:10.1016/S0361-9230 (01)00646-3

Winkelman, M. J. (1992). Shamans, priests, and witches. A crosscultural study of magico-religious practitioners. Tempe, AZ: Arizona State University.

Winkelman, M. J. (2002). Shamanism and cognitive evolution. Cambridge Archaeological Journal, 12(1), 71-101. doi:10. 1017/S0959774302000045

Winkelman, M. J. (2004). Spirits as human nature and the fundamental structures of consciousness. In J. Houran (Ed.), From shaman to scientist essays on humanity's search for spirits (pp. 59-96). Lanham, MD: Scarecrow Press.

Winkelman, M. J. (2010). Shamanism: A biopsychosocial paradigm of consciousness and healing. Santa Barbara, CA: ABCCLIO.

Winkelman, M. J. (2011). A paradigm for understanding altered consciousness: The integrative mode of consciousness. In E. Cardeña \& M. J. Winkelman (Eds.), Altering consciousness multidisciplinary perspectives (pp. 23-44). Santa Barbara, CA: Praeger.

Winkelman, M. J. (2017). Mechanisms of psychedelic visionary experiences: Hypotheses from evolutionary psychology. Frontier in Neuroscience, 11, 539. doi:10.3389/fnins.2017. 00539

Winkelman, M. J., \& Baker, J. R. (2016). Supernatural as natural: A biocultural approach to religion. New York: Routledge. 